\title{
Obturation d'une brèche des digues de l'Isère, lors de la crue de juin 1948
}

\author{
Closing a breach in the Isère dyke \\ after the flood of June 1948
}

\author{
PAR A. RAYNAUD
}

INGENIEUR AU IABORATOMRE DAUPHINOIS D'HYDHAUIIQUE

\begin{abstract}
Obturer une breche en eau courante est un probleme ancien et difficile. Il n'y a pas nne solution universelle, ce sont toujours des cas particuliers oit la nature des fonds et celle des matériaux disponibles jouent un rôle déterminant. - Dangers et limitations du remblayage it lavancement; difficultés du remblayage par couches horizontales. - La rupture des digues de l'lsère (France) en 19:8 : dispositions topographiques; trabaux préliminaires; mise an point sur modèle réduit d'une méthode d'obturation de la breche (longueur: $120 \mathrm{~m}$; fonds : $2 \mathrm{~m} \dot{a} 4,00 \mathrm{~m}$; vitesse: $2,5 \mathrm{~m} / \mathrm{s})$. - Les traman. sur place; leur cadre psychologique, leur rythme.
\end{abstract}

Chaque jour plus recherchée, mieux maîtrisće, mieux asservie, l'eau demeure un redoulable adversaire; apparemment vaincue après des années ou des siècles de lutte acharnéc, elle a parfois de terribles sursauts, et l'Homme, l'Ingénieur, voil se retourner contre lui les armes mèmes qu'il avait si péniblement forgées. Or, dans le grand arsenal de l'Hydraulique, la digue oceupe peutêtre la première place et la plus ancicnne. Est-il un ouvrage qui soit plus intimement mêlé à l'histoire de nos eivilisations? De la solidité de ce rempart dépendent souvent la vie de centaines de milliers d'hornmes, la prospérité d'immenses territoires. Symbole de sécurité, symbole de richesses lorsqu'elle «tient 》, la digue qui erève sous la poussée des eaux peut provoquer de véritables cataclysmes, dont certains appartiennent désormais à l'histoire.

\begin{abstract}
To close a breach in flowing water is an old and still difficult problem. There is no universal solution, each case is particular principally because of important role played by the nature of the bed and the material available. Dangers and limitations of filling progressively; difficulties of filling in horizontal layers. The ruptare of the Isere dykes (France) in 19:8: topographical situation; preliminary worls; denelopment on a scale model of a method of closing the breach (length: $120 \mathrm{~m}$; depth: $2 \mathrm{~m}$ (o) $4,5 \mathrm{~m}$; nelocily: $2,5 \mathrm{~m} / \mathrm{s})$. The works on site; their psychological framework, theit rhythm.
\end{abstract}

L'exemple que nous rapportons ci-aprés est certes assez modeste vis-i-vis de ces grandes catastrophes, vis-à-vis, pour prendre un exemple récent d'une nature peu difrérente d'ailleurs, des inondations qui ont ravagé la Hollande et les còtes de la mer du Nord il y a quelques mois seulement. Cependant, de nos études, de nos essais, de l'expérience que nous avons acquise à cetle occasion, il nous semble possible de dégager quelques réflexions susceptibles d'aider à la mise au point de certains éléments de doctrine concernant le colmatage d'une brèche dans une digue en eau courante. Les "recettes " dans ce domaine ne manquent évidemment pas, elles sont aussi anciennes que le mal. Il semble bien, en particulier, que le peuple chinois soit le premier à avoir su organiser la lutte à une échelle que situe parfaitement le gigantesque Fleuve Jaune. 
Nest-ce pas, dit-on, en luttant contre les méfaits du Fleuve Jaune que, plus de 2.000 ans avant Jésus-Christ, Ye le Grand devint un héros national et le fondateur de l'une des grandes dynasties : celle des « Hia ». Et le corps des ingénieurs chinois qui, aujourd'hui encore, a pour seule mission de surveiller, d'entretenir, d'améliorer et, éventuellement, de réparer les ouvrages de défense, date très probablement de cette époque. Il n'y a pas très longtemps encore, en 19371938 , le Fleuve Jaune creva ses digues et provoqua l'une des plus grandes inondations de son histoire. Les Ingénieurs chinois firent la preuve qu'ils avaient su mettre à profit ces siècles d'expérience, non sans exploiter au maximum tous les moyens de leur temps.

Malheureusement, les textes chinois, du moins ceux qui nous sont parvenus, ne sont guère prolixes à ce sujet; dans une littérature plus pro('he et plus accessible, c'est du début du xvin' siècle que datent, à notre connaissance, les premières publications faisant état dans leurs grandes lignes des méthodes d'obturation de brèches, telles que nous les concevons aujourd'hui. Nous voulons parler de l'ouvrage du capitaine J. Perry : «An account of the stopping of Daggenham breach » (Printed for Benj. Tooke of the Middle Temple Gate in Fleet Street, and saled by J. Peele at Lock's Head in Pater-NosterRow; London, 1721). Plus près de nous, enfin, grand nombre de travaux contemporains ont mis en évidence, chacun dans un certain domaine, quelques principes valables dans les cas particuliers envisagés. Ce sont par exemple :

- Certains travaux de réfection des digues de Hollande, notamment ceux de la digue de Valkeren;

- Les travaux de l'ingénieur russe M. Isbash qui a particulièrement étudié les formations de batardeaux en matériaux lancés dans les courants. Signalons que les très intéressants travaux de cet ingénieur ont été repris et étendus par M. C. BLANCHET (cf. la Houille Blanche, $\mathrm{n}^{\text {os }} 2$ et $6-1946, \mathrm{n}^{\circ} 1$ 1947);

- Les travaux de réfection des digues du Colorado, lors de la rupture cẻlèbre qui provoqua dans la basse plaine de l'Imperial Valley, située au-dessous du niveau de la mer, un lac artificiel dont on voit encore les vestiges.

Ce qui ressort le plus nettement de ces diverses expériences est l'infinie diversité des problèmes posés. La difficulté vient, pour une part, de ce qu'il n'existe guère de règle générale valable dans tous les cas : chaque fois, il convient d'examiner minutieusement les conditions particulières dans lesquelles on se trouve placés, chaque fois les moyens à mettre en œuvre, le minulage des opérations doivent ètre mis alu print en fonction des données locales, et lout spécialement :

- De la nature des fonds de la brèche (sable, gravier, rocher, etc.);

- Des matériaux dont on peut disposer commodément et en quantité suffisante pour les travaux.

Cependant, quelques recommandations d'ordre tout à fait général peuvent, semble-t-il, être avancées.

Il y a toujours intérêt, en cours de travaux, à éviter à chaque instant l'apparition, dans une zone quelconque, de vitesses susceptibles de compromettre l'équilibre des matériaux utilisés, ou mème de nécessiter la mise en cuvre d'un volume de matériaux bien supérieur à ce qu'exigeaient les vitesses initiales dans la brèche.

Ainsi, sur des fonds sableux et profonds, il serait maladroit de déverser en premier stade de gros blocs en vrac. En effet, dans cette hypothèse, au fur et à mesure que le massif tendrait a se développer, l'augmentation de la dénivelée entre les plans d'eau amont et aval accroîtrait les vitesses de circulation dans le massif. Ce phénomène favoriserait les affouillements autour des gros blocs reposant sur le fond : ces blocs s'enfonceraient alors, se faisant littéralement happer par le fond sableux. De toute évidence, une telle méthode mal adaptéc aux conditions locales exigerait beaucoup plus de matériaux qu'une méthode plus rationnelle. Dans de semblables circonstances, il serait prudent, avant le déversement des matériaux, de réaliser sur le fond sableux un pavage de fascines lestées par exemple.

Dans le même ordre d'idée, et comme l'ont bien montré le capitaine PERry et beaucoup d'autres après lui, on peut éventuellement, au début des travaux de réfection (si la chose est possible) commencer le remblayage de la brèche à l'avancement à partir des deux extrémités, mais, dès que l'accroissement corrélatif des vitesses de l'eau est tel que :

- Le fond mobile de la brèche s'affouille;

- Compte tenu des matériaux utilisés (fond constitué d'éléments plus gros que ceux utilisés pour obturer la brèche), le massif de remblayage nécessite un rechargement important;

il faut stopper le remblayage à l'avancement pour procéder comme nous le détaillons plus loin, en faisant progresser la crête de la digue en cours de réfection le plus horizontalement possible.

Les quatre photographies ci-contre, dues à la courtoisie du professeur SHEN YI (Economic Commission for Asia and the Far East. Unit-Na- 
Ce bouchon une fois réalisé, il ne reste plus quà le laisser tomber dans la brèche, ce qui est obtenu très simplement en tranchant simultanément tous les câbles à un signal donné par le chef de chantier. C'est la fin de cette dernière operation qu'illustre la derniere des photographies (fig. 4).

Tout à fait significalives, quant à la technique adoptée, ces photographies montrent également qu’à défaut de moyens matériels très puissants, l'ingéniosité permet de venir à bout de difficultés apparemment très lourdes.

Les travaux que nous allons relater maintenant sont certes assez anciens puisqu'ils datent de 1948. Les circonstances ne nous onl pas permis de fatre cette publicalion plus tôt, mais ceci n'enleve rien, pensons-nous, à la valeur du modeste appoint que nous espérons apporter à l'amélioration d'une technique qui remonte aux sources les plus lointaines des civilisations.

\section{I. - APERÇU TOPOGRAPHIQUE DU LIEU DE LA RUPTURE}

Pour mieux situer le problème faisant l'objet de cet article, precisons que la rupture de digue dont il s'agit se situe dans le Bas-Grésivaudan un peu à l'aval de Grenoble, au droit du bec de l'Echaillon.

Dans la zone de la rupture principale, l'Iscire fait un coude et contourne le Bec de l'Echaillon (fig. 5); a ce nivau et parallelement à l'Isère, coule le canal de Vence dans lequel vient se jeter le ruisseau du Palluel. La digue rive droite de l'Isere tient lieu de digue rive gauche du canal de
Vence; coté plaine, le canal est limité par une deuxieme digue, arasée sensiblement, dans la zone qui nous intéresse, à Ja même cole que la digue Isère.

Lors de la crue de juin 1948, l'Isere creva sa digue rive droite, en différents points, bien à l'amont de la zone qui retiendra notre altention. Les eaux de ces breches grossirent anormalement le canal de la Vence qui rongea jusqu'à l'effondrement la digue Isère, juste au niveau du bec de l'Echaillon; a ce moment, le flot franchit la di-

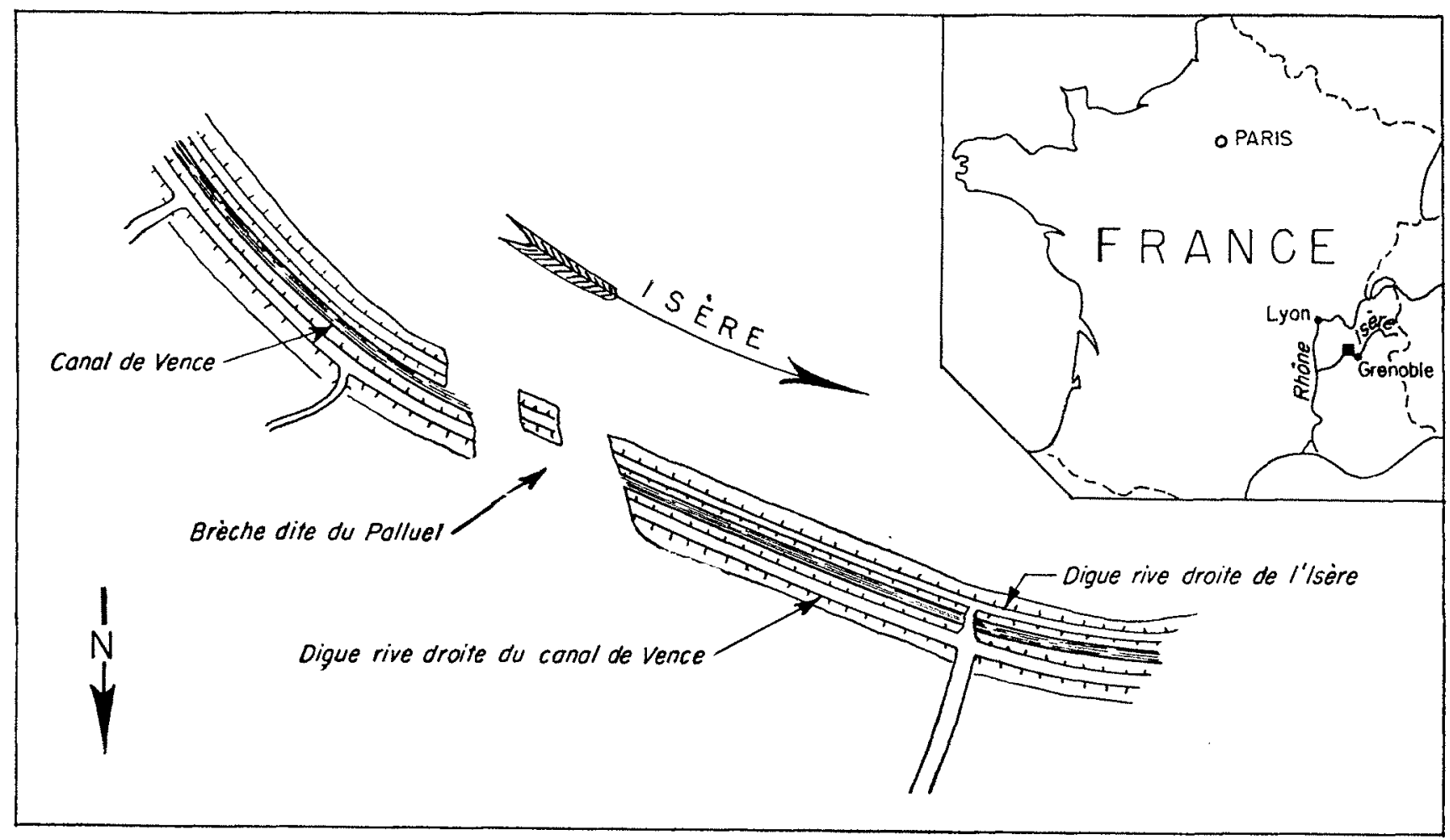




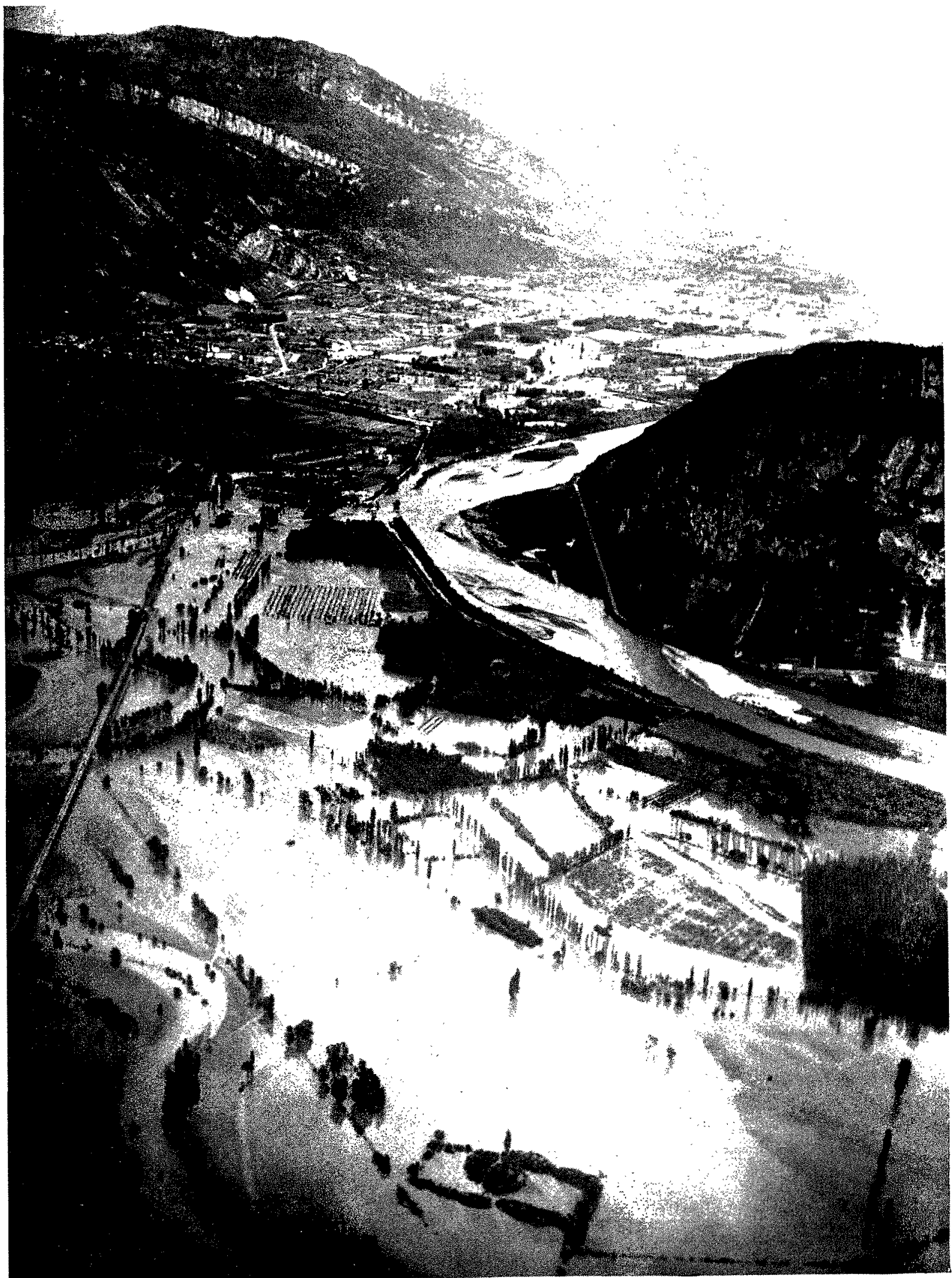

Garbe Photopress

Fig. 6. - Vue aérienne de la brèche dite du Palluel, prise de l'aval vers lamont de lisere. 
gue rive droite du canal de Vence, qui, non perrayée du côté plaine, fut rapidement emportée.

On se trouva done loealement en présence de la situation suivante (fig. 5 et 6 ) :

Lat digue rive droite de l'Isère, rompue en deux endroits différents, sur une longueur tolale de l'ordre de $250 \mathrm{~m}$;

- La digue rive droite du canal de Venee, rompue sur une longueur de l'ordre de $120 \mathrm{ml}$ avec des profondeurs minima, ver's le milieu, de $4,5 \mathrm{~m}$.

Quelques jours après le maximum de la crue, on peut évaluer que les deux tiers du débit de I'Isère allaient se répandre dans la plaine par cette brèche largement ouverte.

Pour diverses raisons (facilités d'accès, concentration plus commode des travaux...), que nous n'examinerons pas ici, il fut décidé de procéder à lobturation en plein courant de la brèche cxistant dans la digue rive droite du canal de Vence.

Cette brèche, d'une longueur totale de l'ordre de $120 \mathrm{~m}$, avait des fonds allant de $2 \mathrm{~m}$ à $4,5 \mathrm{~m}$ et en certains points des ritesses alteignant $2,5 \mathrm{~m} / \mathrm{s}$.

Les travaux sur place furent effectués par les Entreprises Pascal, de Grenoble, sous la direction de M. l'Ingénieur en chef PIÉrR, assisté de M. Roller, ingénieur des Ponts et Chaussées, et de MM. Mathrev et Darbon, ingénieurs des Traraux Publics; enfin, des les premiers jours, les travaux furent suivis par le Laboratoire Dauphinois d'Hydraulique des Etablissements Neyrpic, qui firent une étude rapide sur modele réduit, dans le but de broposer et de contróler les solutions techniquenent satisfaisantes et susceptibles d'être retenues.

Enfin, nous nous permettons de profiter de cette occasion pour remercier M. Donges, secrétaire général honoraire du Ministère des Traraux Publics et des Transports, pour les bienveillants conseils et les précieux encouragements qu'il a bien voulu nous prodiguer tout au long de ces opérations, que son attachement à la région grenobloise, et le souvenir de ses propres travaux sur l'Isère, lui ont fait suivre avec unc toute particulière attention.

\section{II. - TRAVAUX PRELIMINAIRES EFFECTUES SUR PLACE DURANT L'EVOLUTION DE L'ETUDE SUR MODELLE REDUIT}

Les ruptures de digues se produisirent le 23 juin. Dans la période du 24 juin au 4 juillet, on procéda à la construction de chemins d'accès a l'échelle des travaux. Pendant ce temps, les premiers essais sur modèle étaient entrepris, permettant de s'orienter très rapidement vers la solution : obturation par jets de gabions Palvis dans le courant. Sur place, l'exécution de la passerelle de lancement commençait aussitôt.

Cette passerelle, dont les dispositions correspondaient finalement au mode de lancement retenu des gabions, est posée sur 28 palées, distantes de $4 \mathrm{~m}$ les unes des autres, parallèles au courant et inclinées relativement à la crête de la digue.

\section{Chaque palée est constituée (fig. 7) :}

- D'une série de 3 pieux de $9 \mathrm{~m}$ fichés de $3 \mathrm{~m}$, distants de $2 \mathrm{~m}$, moisés et contreventés entre eux, et prévus pour supporter deux voies de $0,60 \mathrm{~m}$ :

- Enfin d'un quatriime pieux dans le prolongement des trois premiers, et a $5 \mathrm{~m}$ a l'aval des troisiemes; ces quatriènes pieux de chaque palće sont moisés entre eux et reliés chacun anx trois pieux les précédant par des bois amovibles devant maintenir l'écartement de $5 \mathrm{~m}$.

Le battage des pieux se fit à l'aide d'une sonnette équipée d'un mouton de $400 \mathrm{~kg}$, le toul monté sur une portière constituée par deux batardeaux du Génie Militaire.

Comme nous le précisons par la suite, les dispositions particulières retenues pour la passerelle permettaient de laisser choir commodément dans le courant des gabions de $4 \mathrm{~m}$ de longueur sur $1 \mathrm{~m}^{2}$ de section, leur plus grande longueur parallèle au sens du courant. Exceptionnellement, à l'amont d'un certain nombre de pieux, on convint de lancer des gabions de $3 \mathrm{~m}$ de longueur seulement.

Avant de lacher des gabions dans le courant, une première élape consiste à consolider la levre rive droite de la brèche, qui au début des travaux était la seule commodément accessible 


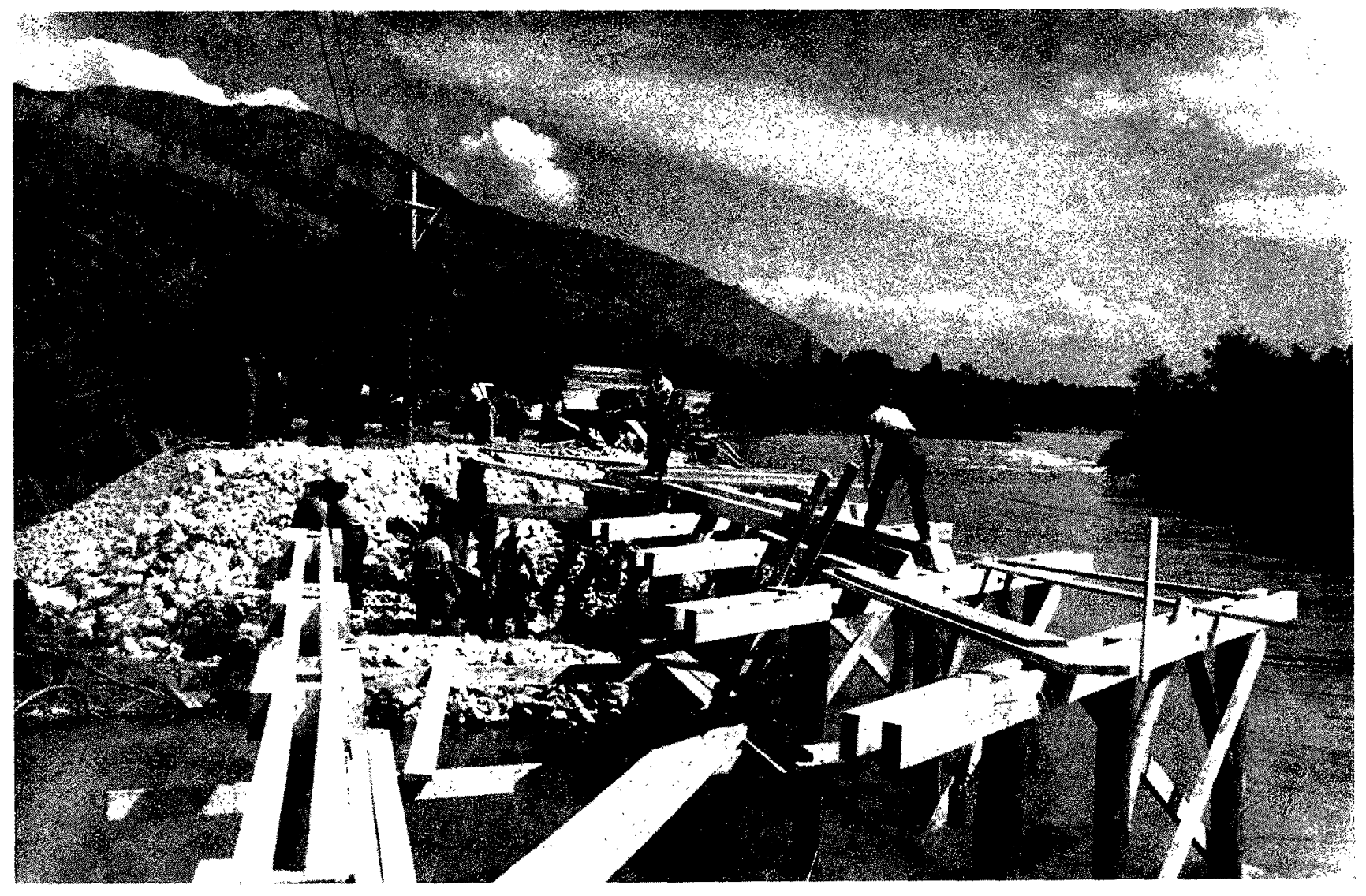

Frg. 7. - Passerelle de lancement des gabions en cours de construction.

Cliche Dal-Bon.

(pour la lèvre rive gauche, les conditions d'accès nécessitaient en effet que la passerelle soit entièrement terminée).

Celte consolidation locale se fit à partir du 4 juillel par basculement sur place de gabions et par empilement de matériaux. Une fois cette consolidation partielle terminée, la construction de la passerelle n'étant pas encore suffisamment avancée, on commença la digue d'avancement, sur une longueur totale de $15 \mathrm{~m}$, ce qui constituait finalement un épi de protection de la levre rive droile de la brèche.

Le 7 juillet commencait l'échouage des gabions a partir de la passerelle.

\section{III. - ESSAIS SUR MODELE REDUIT}

La décision ayant été prise d'obturer la brèche par jets de gabions dans le courant, il s'agissait alors de s'entourer du maximum de sécurité dans l'exécution des travaux sur place et de définir un certain nombre de conditions techniques essentielles.

Fn lout premier lieu, il s'agissait de savoir s'il était préférable de lancer les gabions, leur plus grante longueur perpendiculaire ou parallele au sens du courant; cette donnée était d'autant plus urgente qu'elle conditionnait en même temps les formes et dispositions de la passerelle de lancement.

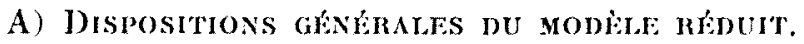

Les essais ont élé réalisés rapidement sur un modele réduit existant, aménagé pour la circonstance.

Il pouvait être considéré comme à l'échelle du 
1/10, et correspondait aux caractíristiques suivantes :

-- Briche représenté sur une longueur totale de lordre de $20 \mathrm{ml}$.

-.. Levres de l'elément de briches en dur (il ne s'agissait d'ailleurs pas d'étudier sur ce modele le comportement éventuel de la liaison corps de la digue-liveres de la brèche).

-- Fond affouillable, au droit de la breche.

Enfin, de manière à reproduire plus fidèlement la nature, à l'amont de la brèche nous avions sur le modèle un long déversoir latéral qui permettait, dans la mesure où le lancement des gabions clevait le plan d'cau à l'amont, de diminuer automaliquement le débit passant par la breche.

Le modèle élait de plus équipé :

- A lamont, d'un déversoir permetlant de mesurer les débits;
-- D'un volet aval permettant de régler la position du plan d'eau dans la plaine, à l'aval de la breche;

- De deux pointes limnimétriques, l'une à l'aval, l'autre à l'amont de la brèche.

B. - Premere sénte d'essais. -- Echouage des GABIONS, LEUR PLUS GRANDE LONGUEUR PERPENDICULAIRE A LA DIRECTION MOYENNE DU COURANT.

La passerelle de lancement est disposce $2 \mathrm{~m}$ au-dessus du plan d'eau initial. Avant d'être cohoués, les gabions sont disposés sur la passerelle et sont ensuite basculés sans vitesse initiale dans le courant.

- La profondeur d'eau maximum au droit de la breche est de l'ordre de $4,5 \mathrm{~m}$;

-. Les fonds sont afrouillables.

Le Lableau I précise le délail d'un des essais type réalise sur le modèle.

TABLEAL I

\begin{tabular}{|c|c|c|c|c|c|c|c|c|c|c|c|c|c|}
\hline $\begin{array}{l}\text { Instants d'échouage des } \\
\text { gabions sur le modele.. } \\
\text { (min.) }\end{array}$ & $0+\varepsilon$ & $5+\varepsilon$ & $12+\varepsilon$ & $20+=$ & $28+\varepsilon$ & $35+s$ & $45+\varepsilon$ & $54+\varepsilon$ & $63+\varepsilon$ & $73+\varepsilon$ & $83+\varepsilon$ & $90+\varepsilon$ & $95+\varepsilon$ \\
\hline $\begin{array}{l}\text { Nombre de gabions } \\
\text { echonés } \ldots \ldots \ldots \ldots\end{array}$ & 7 & (i & 9 & $\overline{5}$ & 7 & 7 & 7 & y & 9 & 9 & 7 & 7 & 10 \\
\hline $\begin{array}{l}\text { Volume de materiaux dé- } \\
\text { versés dans le modole. } \\
\text { (decalitres) }\end{array}$ & 4 & 4 & 4 & 5 & 6 & 5 & 6 & 5 & 5 & 5 & 2 & & \\
\hline $\begin{array}{c}\text { Surélévation du plan } \\
\text { d'eau amont........ } \\
\text { (u nature) }\end{array}$ & $0,0.4$ & 0,07 & 0,13 & 0,21 & 0,27 & 0,34 & 0,43 & 0,50 & 0.52 & 0,56 & 0,62 & 0,67 & 0,74 \\
\hline
\end{tabular}

Les figures 8 et 9 montrent :

- Ia figure 8 , une vue de la portion de briche figurée sur le modìle à l’instant $(45 \mathrm{mn}+\varepsilon)$ du tableau précédenl. Toute une rangée de gabions altend l'instant 54 mn pour être à son tour basculce dans le courant, gabion par gabion.

-_a figure 9, une vae du modele pratiquement a sec, une fois l'essai terminé.

Nous reviendrons plus loin sur les conclusions qu'il $y$ a lieu de tirer de cet essai.

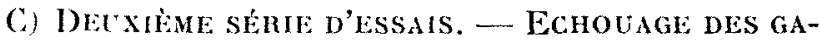
BIONS, LEUR PLUS GRANDE LONGUEUR PARALLELE A IA DHECTION MOYENNE DU COURANT.

Les conditions hydrauliques de ce type d'essais sont identiques aux conditions du type d'essais précédent, avec cette différence toutefois que :

-- Les levres non affouillables de la breche sont plus larges que précédemment dans le sens de l'écoulement.

- Nous avons figuré sur le modele les pieux de la passerelle de lancement, distants de

(*) Au cours de l'essai, de maniere a oblurer les interstiees pourant subsister cntre deux gabions adjacents ou un gabion et le fonds affoubllable, nous deversions a l'amont de lélement de diguc en formation un certain nombre de seaux de 10 litres de malériaux tout renant. 


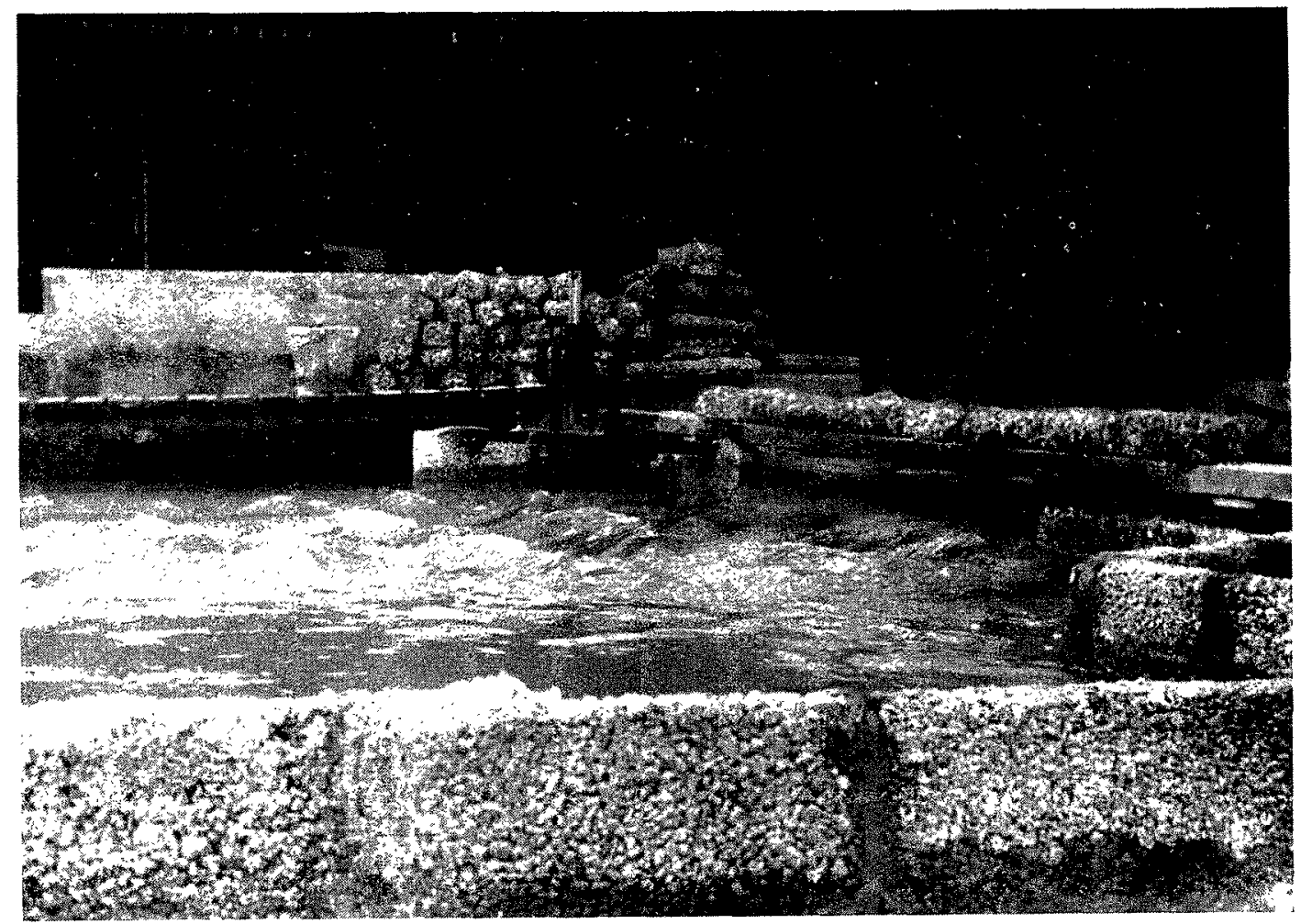

Fig, 8. - Modele en cours d'essais. Toute une rangée de gabions est sur le point d'ètre basculée dans l'écoulement.

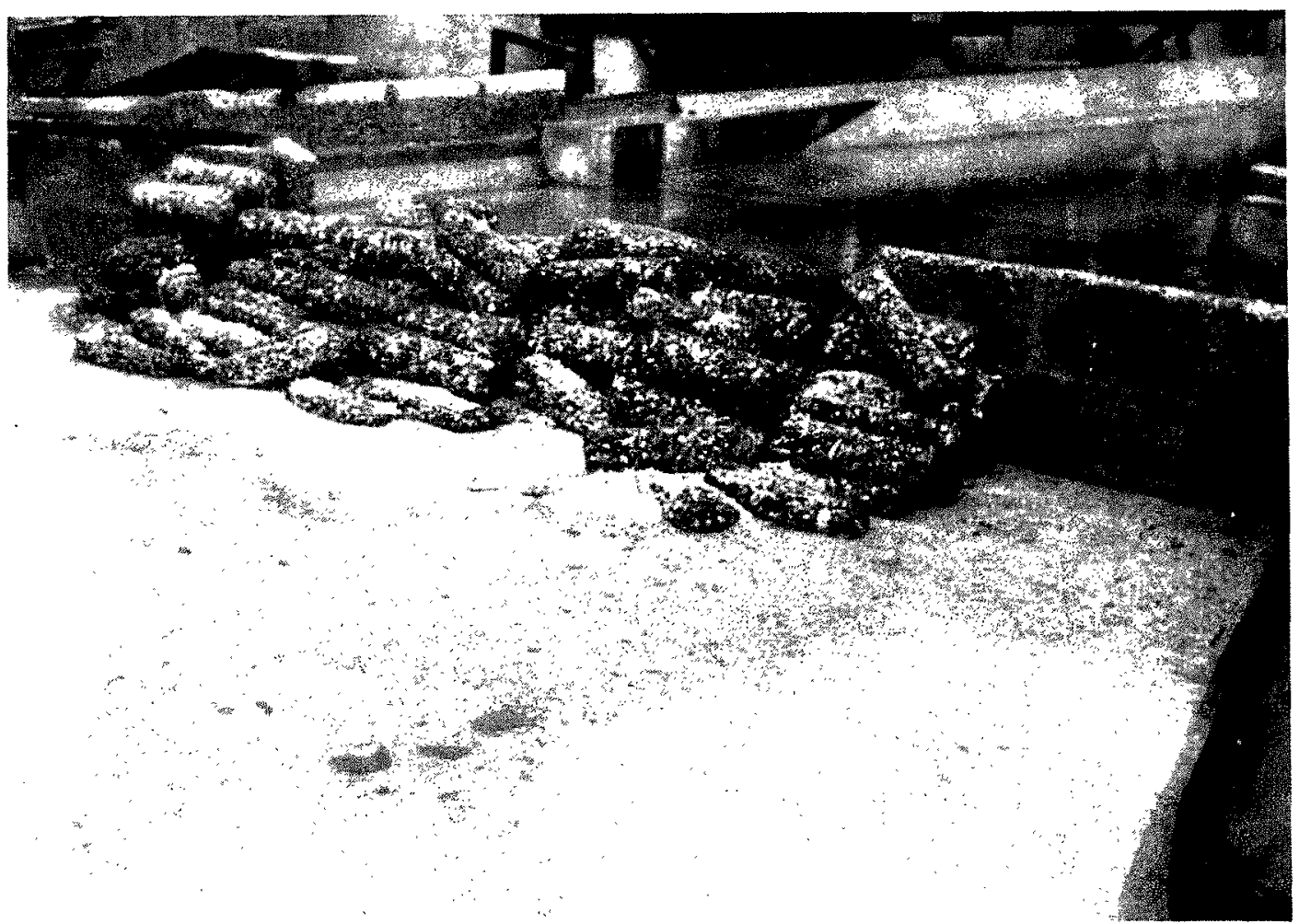

Frg. 9. - Modèle a sec aprés un essai. Remarquer l'aspect hétérogene du massif résultant du mode d'échouage des gabions (leur plus grande longueur perpendiculaje a la direction moyente du courant). 


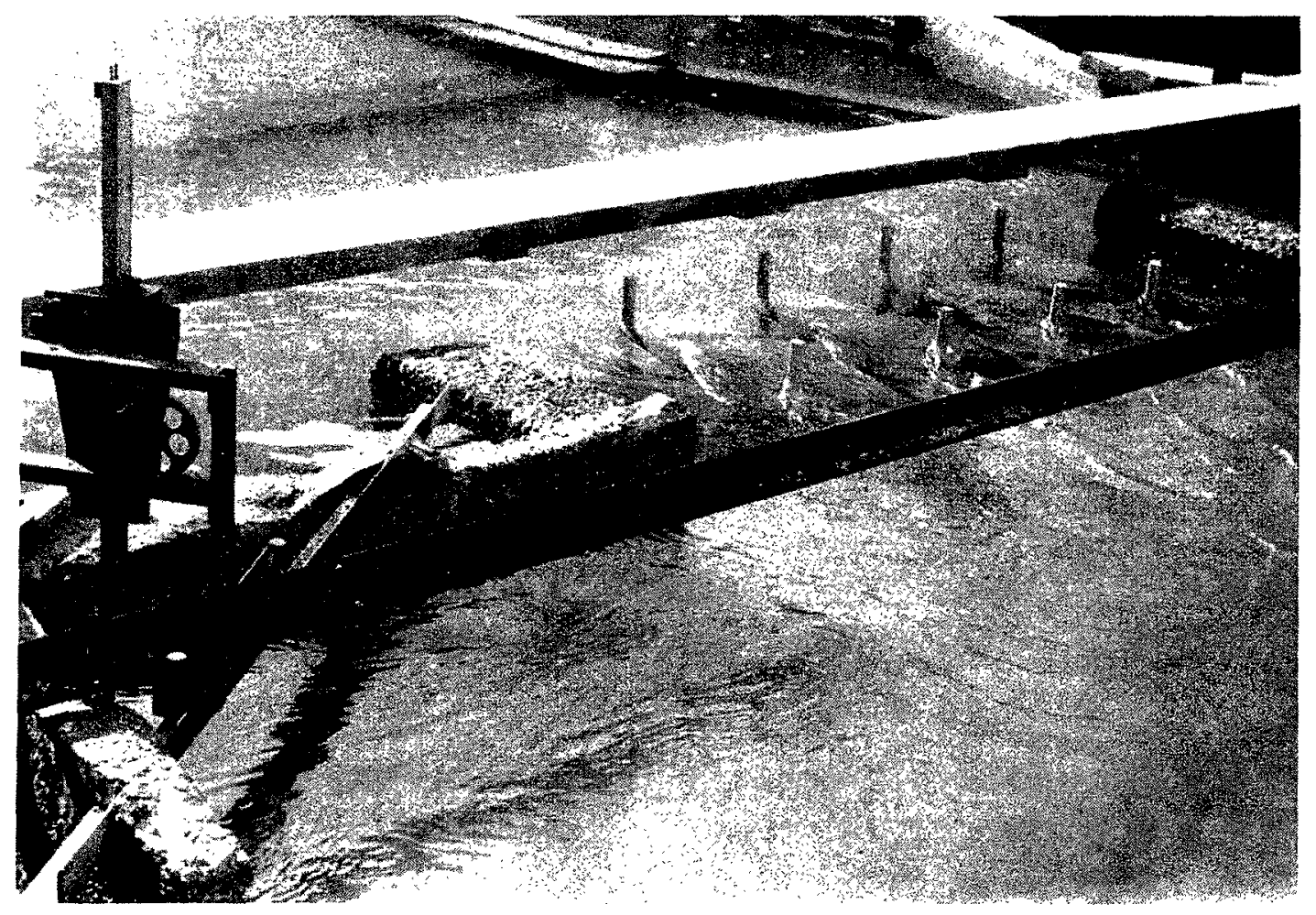

Firi. 10. - Modele en cours d'essais à l'instant $30 \mathrm{mn}$

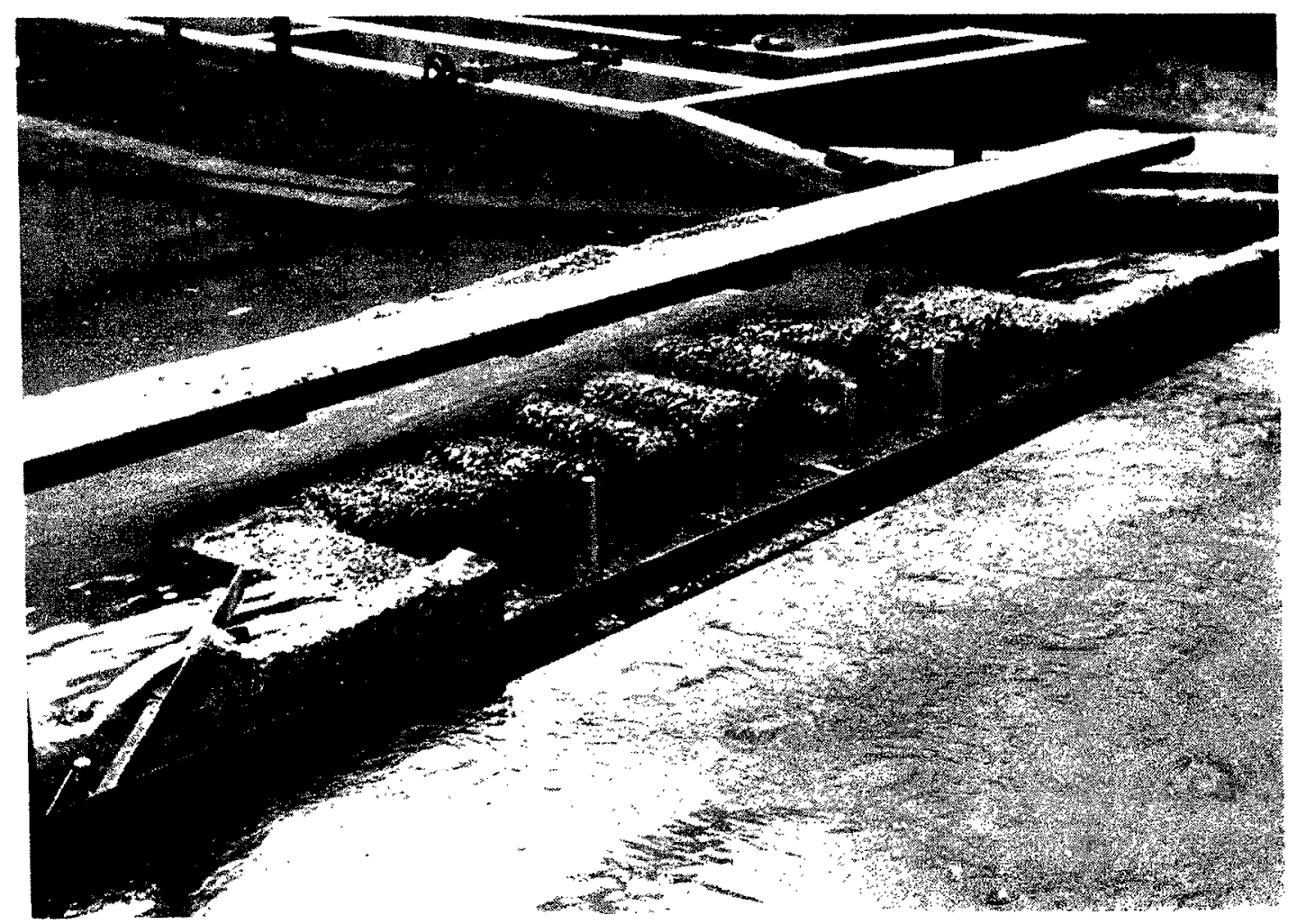

Fir. 11. - Vue du modèle en fin dessai; les gabions ont été échoués leur plus grande longueur parallèle à la direction moyenne du courant. 
$5 \mathrm{~m}$ l'un de l'aulre dans le sens de l'écoulement.

Les gabions étaient déversés les uns aprés les autres dans le courant à la manière dont ils le seraient dans la nature.

Le tableau II précise le détail d'un des essais type réalisé sur le modèle. De même que dans la

\section{TABLEAU II}

\begin{tabular}{|c|c|c|c|c|}
\hline $\begin{array}{c}\text { Instants d'échouage des } \\
\text { gabions sur le modèle.. } \\
\text { (min.) }\end{array}$ & $0+\varepsilon$ & $15+\varepsilon$ & $30+\varepsilon$ & $45+\varepsilon$ \\
\hline $\begin{array}{c}\text { Nombre de gabions } \\
\text { échoués ........... }\end{array}$ & 19 & 19 & 21 & 30 \\
\hline $\begin{array}{c}\text { Volume de matériaux dé- } \\
\text { versés dans le modéle.. } \\
\text { (décalitres) }\end{array}$ & 4 & 10 & 10 & 18 \\
\hline $\begin{array}{c}\text { Surélévation du plan } \\
\text { d'eau amont.......... } \\
\text { (m nature) }\end{array}$ & 0,11 & 0,27 & 0,42 & 0,6 \\
\hline
\end{tabular}

première série d'essai, nous déversions à l'amont de la digue en cours de réalisation, des seaux de matériaux tout venant.

Les figures 10 et 11 montrent:

- La figure 10 une vue de la portion de brèche figurée sur le modèle à l'instant $(30 \mathrm{mn}+\varepsilon)$.

- La figure 11 une vue du modele une fois lessai terminé.

\section{D) Résultats des issais.}

\section{Première série d'essais.}

Cette première série d'essais a permis de préciser que :

- Les gabions de $4 \mathrm{~m}$ de longueur el de $1 \mathrm{~m}^{2}$ de section, échoués sans vitesse initiale, leur plus grande longueur normale à un courant de $2,5 \mathrm{~m} / \mathrm{s}$ de vitesse, avaient une tendance assez nette à rouler sur le fond sur une certaine distance.

Si ce mode de lancement avait été retenu, dans le but de diminuer la largeur à la base de la digue (et par là même, le nombre de gabions échoués), nous pensons qu'il eût été intéressant de prévoir des dispositions particulières, capables d'arrêter les premiers gabions à une distance raisonnable de leur point de chute. Des dispositions appropriées, faciles à imaginer, consistent par exemple à battre des pieux convenablement disposés et répartis dans le courant.
- Les risques de renard sont peut-etre moins grands pour ce mode de lancement que pour le mode suivant (... 2" série d'essais). Il $\mathrm{y}$ a avantage (surtout dans les premicres séries de lancers) à échouer en quinconce les séries successives de gabions (cf. les trois premiers échouages successifs de la première série d'essais); ainsi le deuxieme lancer tend a obturer les orifices qui auraient pu se former entre les gabions du premier lancer; il en est de mème pour le troisìme, relativement au deuxième, etc.

Les risques de renard subsistent évidemment dans toute leur acuilé si le gabion tombe sur un fond non plan (fig. 12); il peut rester un espace

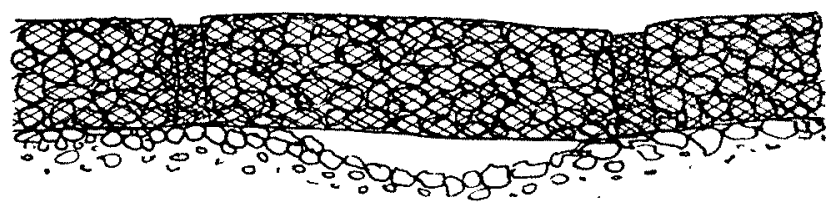

Fis. 12. -... Gabions échoues leur plus grande longucur perpendiculaire al sens du conrant. Risque de fomation d'espace libre entre un gabion et le fond non plane.

libre entre le gabion ef le fond afrouillable, à travers cet espace libre les vitesses augmentent avec la déniveléc totale : plan d'eau amont -.... plan d'eau aval. Il est alors indispensable de jeter dans le courant à l'amont de la digue en formation, dès la premiere rangie de gabions échoués, des matériaux suffisamment gros susceptibles dobturer les espaces libres qui ont pu se former.

II y a lieu de consolider les lives rives droite et gauche) de la briche par des gabions, de maniere à éviler toul élargissement possible au fur et à mesure de la construction du corps de la digue.

- Pour des questions de stabilité et afin de faciliter la poursuite des travaux, il est essentiel d'élever progressivement la crête de la digue le plus horizontalement possible. Les inégalités en altitude ne doivent pas, en principe, dépasser l'épaisseur d'un gabion.

Comme le montre la figure 9, pour le mode de lancer considéré, une fois l'essai terminé, le corps de la digue forme un tout d'apparence hétérogène. Pour ce mode de réalisation il eût été à eraindre des accidents du type suivant:

- Un gabion d'un $n^{\text {tème }}$ lancer provoque le déséquilibre, et la chute sur le parement aval de la digue en cours de formation, d'un gabion du $(n-1)^{\text {tème }}$ lancer, provoquant 
ainsi une breche d'une hatuleur de 2 gatbions.

Il n'était pas exceptionnel d'observer ce type d'accident au cours de nos essais.

\section{Deuxième série d'essais.}

C'est finalement le type de lancement qui a été retenu pour les travaux sur place.

Un certain nombre de remarques sont communes aux deux modes de lancers :

- Nécessité de consolider les levres rives droite et gauche de la brèche.

- Nécessité d'élever progressirement la crêle de la digue le plus horizontalement possible, de manière à éviter de créer une brèche secondaire dans la brèche principale. L'expérience a en effet montré que cette brèche secondaire est toujours difficile à résorber.

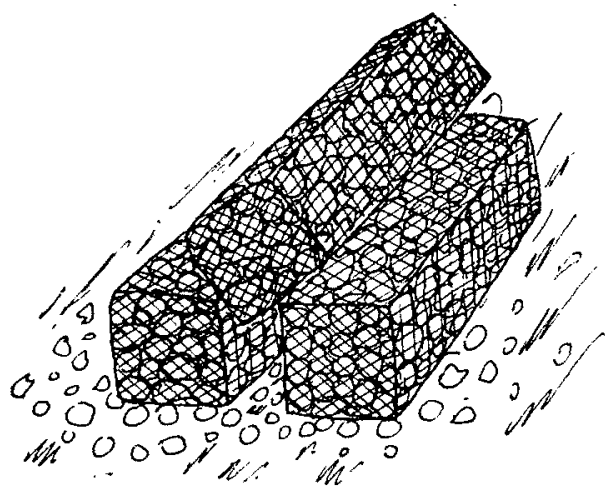

Fia, 13. - Gabions échoués leur plus grande longueur parallèle atu sens du courant. Riscues de formation d'espace libre entre deux gabions.

- Les risques de renard existent toujours dans ce mode de lancer (fig. 13). En admettant une charge de l'ordre de $1,5 \mathrm{~m}$ sur un orifice susceptible de se former entre deux gabions reposant sur le fond affouillable, on voit qu'il faut s'attendre à avoir des vitesses de l'ordre de $5 \mathrm{~m} / \mathrm{s}$ au voisinage $\mathrm{du}$ fond. A moins que les fonds soient constitués de très gros blocs, et, faute de précautions particulières, il faudrait craindre, avec tout ce que cela comporte, des alfouillements aulour des gitbions.

Cette remarque montre qu'il est essentiel de compléter le programme d'échouage des gabions par des lancers de matériaux à l'amont du corps de la digue en formation, matériaux capables d'obturer les intervalles les plus grands, susceptibles de s'ètre lor'més.

$\mathrm{Si}$, après le déversement de la deuxième rangée de gabions, il s'avérail qu'un on plusieurs de ces intervalles aient des dimensions telles qu'ils ne puissent que difficilement s'obstruer à l'aide de blocs commodément utilisables, on pourrait songer à divers artifices tels que :

- Descendre à l'amont de la digue, dans le voisinage de l'orifice, des grillages lestés (par exemple des grillages analogues à ceux utilisés pour les gabions) et procéder ensuite au déversement des matériaux;

- Soit encore lancer des fascines convenablement lestées.

Vers la fin des travaux il convient de rendre étanche le corps de la digue. A cet effet, après que les matériaux les plus gros, susceptibles d'obstruer efficacement les intervalles entre gabions, seront en place, on pourra déverser une succession de couches, chacune étant constituée de matériaux en moyenne plus gros que les intervalles laissés par les matériaux de la couche précédente.

Le critère recherché sera de transformer le corps de la digue en un véritable filtre. A ce propos, pour juger de l'influence des différentes couches -sur l'étanchéité, il y aura lieu d'observer fréquemment les eaux d'infiltration à l'aval de la digue. Dès que ces eaux seront suffisamment claires, on pourra parfaire le colmatage en déversant sur le parement amont des ćléments fins.

Enfin, pour le mode de construction retenu, étant donné, d'une part, l'écartement de $5 \mathrm{~m}$ prévu entre les pieux supportant les travées de lancement et, d'autre part, la longueur des gabions $(4 \mathrm{~m})$, on décida, pour éviter les risques de heurts des pieux aval, de réduire à $3 \mathrm{~m}$ la longueur des gabions lancés dans le courant i l'amont de ces pieux.

\section{IV. - TRAVAUX D'OBSTRUCTION SUR PLACE}

L'échouage des gabions, à partir de la passerelle, commenca le 7 juillet. Pour qu'il pût commencer le plus tot possible, on battit d'abord, sur toute la largeur de la brèche, les trois picux les plus à l'aval de chaque palée, renonçant ainsi à la possibilité de lancer dès le début, et en même temps que les premiers gabions, des matèriaux à l'amont de la digue.

Pendant l'échouage des premiers gabions, on acheva de battre les pieux amont de chaque palée.

Le grillage ayant été préalablement disposé sur 


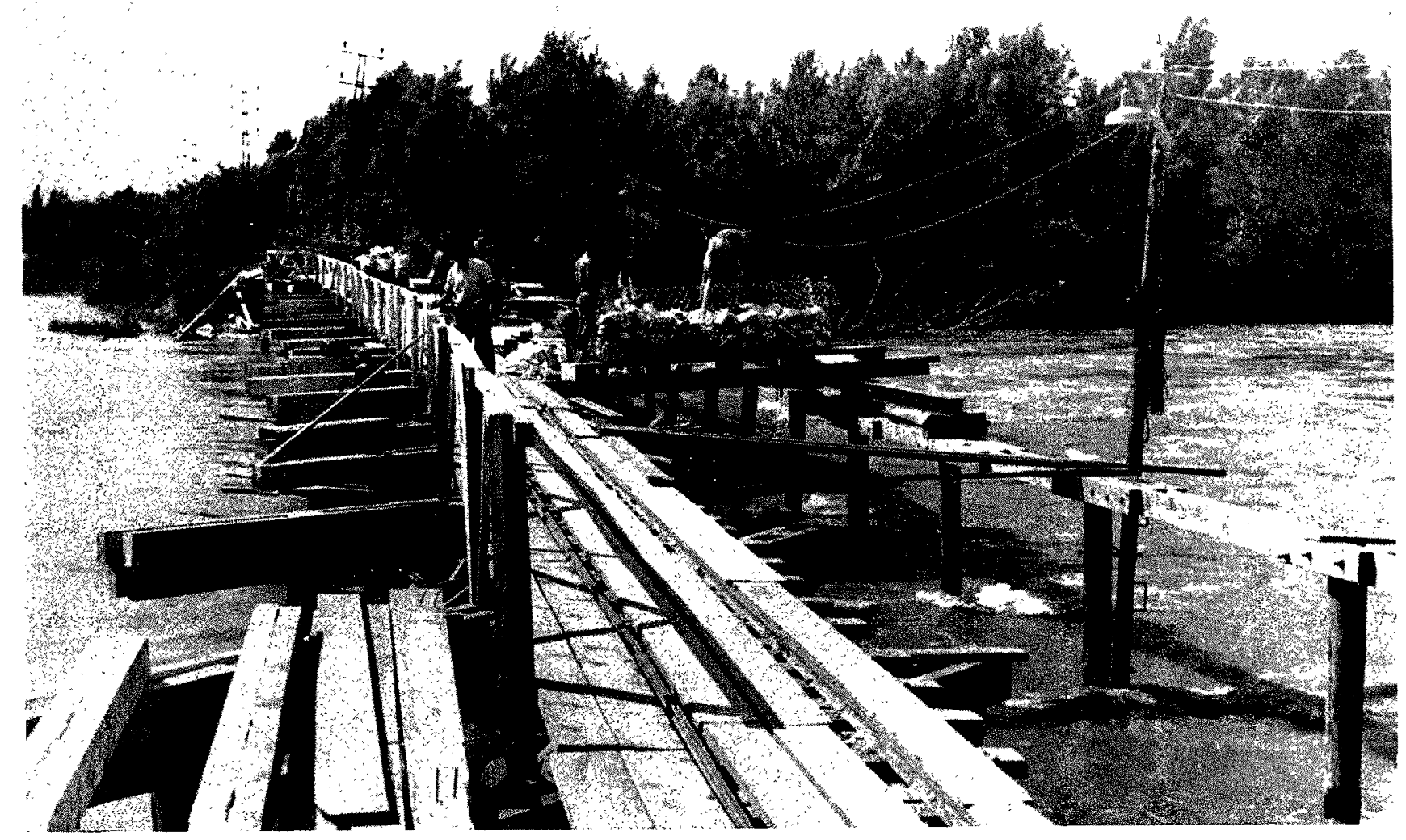

FIG. 14. - Les gabions sont remplis sur place.

la plate-forme de lancement (fig. 14), il est rempli sur place, les matériaux étant amenés par wagonnets sur la voie de $0,60 \mathrm{~m}$. Dès qu'il est plein, le gabion est basculé en plein courant (fig. 15).

Comme les essais en avaient montré tout l'intérêt, les travaux furent menés de manière à élever la crête de la digue le plus horizontalcment possible, malgré les inégalités de cote du fond.

Dès le 10 juillet, date de l'achèvement de la passerelle, on passa à la fixation de la léve rive gauche de la brèche. De plus, la deuxième voie de $0,60 \mathrm{~m}$ étant alors termine, on commenca à déverser dans le courant, à l'amont de la digue en formation, de gros blocage, de manière à obturer les vides pouvant subsister entre les gabions.

L'échouage des gahions se poursuivait ensuite sans coup férir et le $1^{\text {er }}$ août on posait sur le corps de la digue le dernier gahion immergé. L'obluration totale de la brèche pouvait alors être considérée comme terminée. De plus, des le 29 juillet, grâce à un chemin de $5 \mathrm{~m}$ de large constitué sur la digue, on pouvait deverser direetement par camions entiers :

Fig, 15. - Ine fois rempli, le gabion est bascule dans te courant.

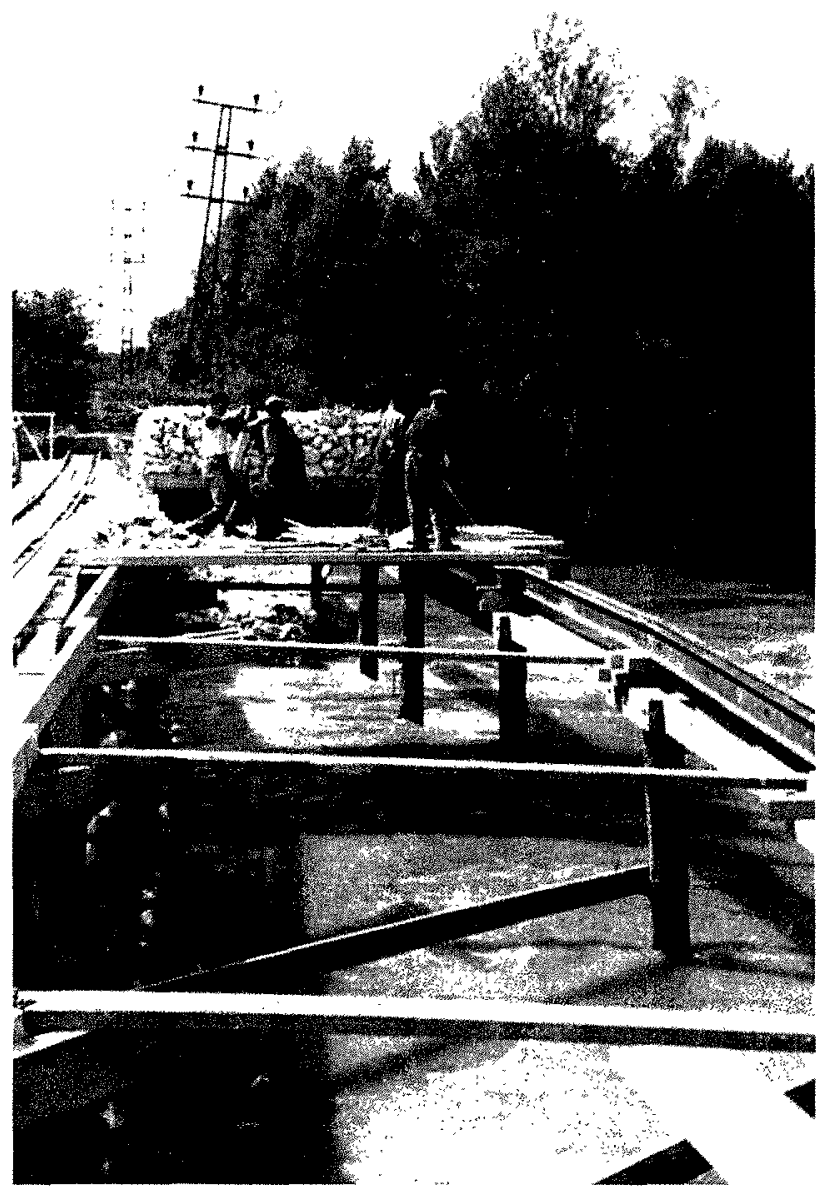


- Du côté de l'Isère: du tout-venant de 0 à $12 \mathrm{~mm}$;

- Du côté de la plaine, du remblai provenant d'un torrent voisin.

A ce moment, la digue est à 1,2 $\mathrm{m}$ aut-dessous de son niveau définitif. Tel que, le corps de la digue est loin d'être ćtanche et un débit d'infiltration relativement important passe au travers.
L'étanchéité linale est réalisée avec beaucoup de bonheur en déversant à l'amont des camions entiers de mâchefer.

Dans cette période, plusieurs crues successives inquiétèrent un certain temps les responsables des travaux, mais finalement on put élever à temps la digue jusqu'à sa cote normale et éviter tout débordement. La photo de la figure 16 montre la digue du côté Isère en cours d'achèvement.

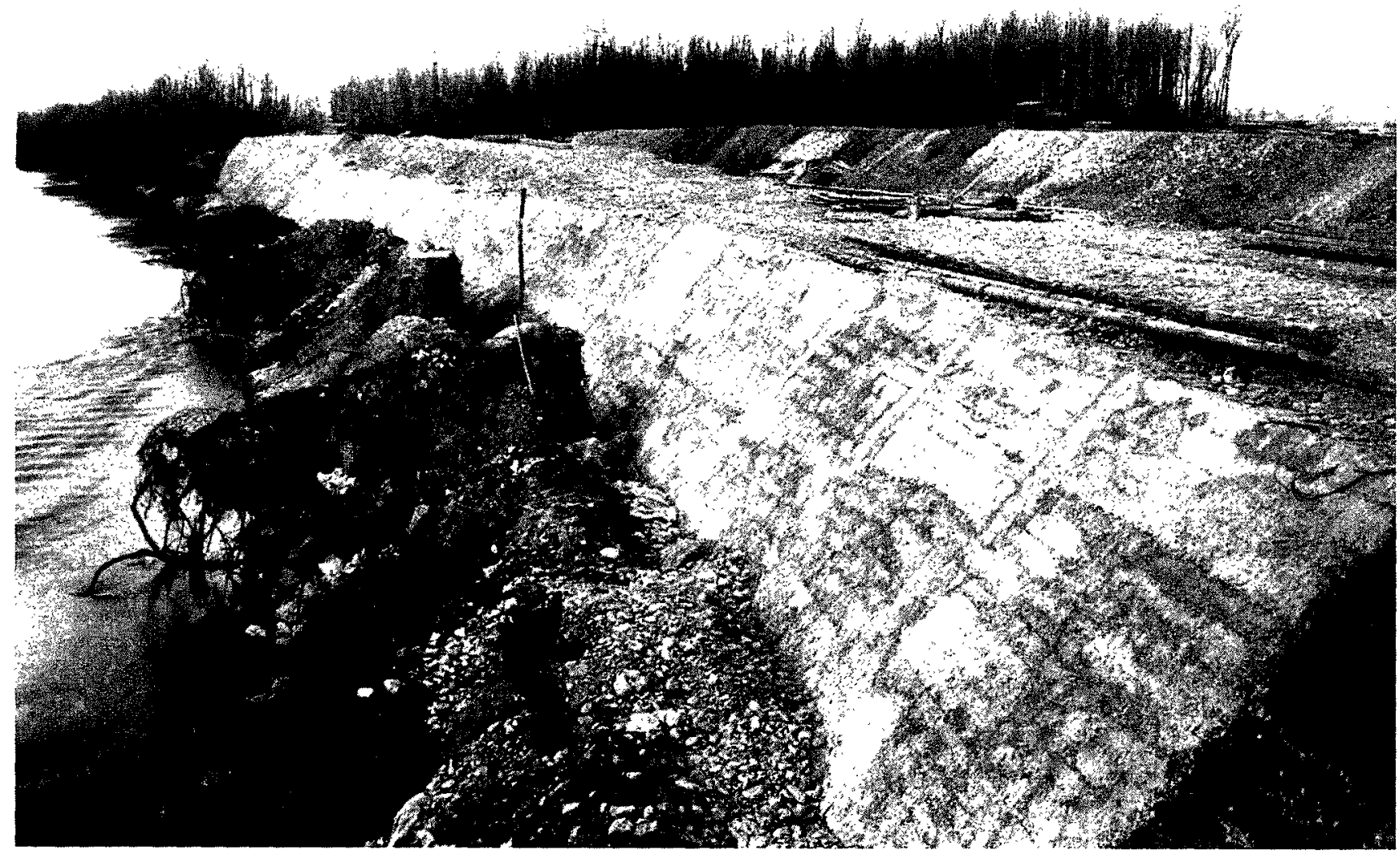

Fici 16. - Digue terminée, vue du côté Isère.

Cliché Photopress. Les quelques matériaux disposés sur l'ourage en montrent l'échelle.

\section{V. - CONCLUSION}

La réfection de ce tronçon de la digue de l'Isère, malgré des conditions de travaux souvent difficiles et des crues successives, particulièrement gênantes et dangereuses, a finalement été une réussite complète.

Elle a montré l'intérèt que pouvait présenter l'utilisation de gabions lancés en plein courant, leur plus grande longueur parallèle à la direction générale de l'écoulement; enfin elle a mis clairement en évidence l'utilité du modèle réduil permettant rapidement de prendre des décisions avec le maximum de sécurité technique.

A titre documentaire, signalons que les tra- 
vaux d'obturation de la hreche du Palluel ont nécessité :

$$
\begin{array}{cl}
2.000 \mathrm{~m}: & \text { de gabions, } \\
7.500 \mathrm{~m}^{*} & \text { de blocage, } \\
400 \mathrm{~m}: & \text { de mâchefer, } \\
38.000 \mathrm{~m}^{*} & \text { de remblais, } \\
800 \mathrm{lonnes} \text { denrochements, } & \\
1.500 \mathrm{~m}^{2} & \text { de perre maçonne. }
\end{array}
$$

Nous terminerons enfin cet expose par une remarque d'ordre psychologique inhérente à la technique mème de la méthode utilisée pour ces travaux.

Dans l'hypothese d'une catastrophe du genre de celle évoquée, les sinistrés viennent fróquemment sur les lieux observer comment se font les travaux et où en est leur état d'avancement. La méthode adoptée qui, sur le plan technique, compte tenu de l'importance de l'accident et des difficultés de réalisalion, s'est avérée excellente, présente l'inconvénient psychologique de nécessiter une préparation relativement longue (batlage des pieux de la passerelle) sans qu'on puisse jeter quelques cailloux dans fo hut de limiler le débit allant vers la plaine, el donner ainsi l'impression que la situation s'améliore progressivement.

Pour le profane, beancoup plus dynamique semhlerail me méthode plus brutale qui consisterait par exemple a essayer de rétablir la digue a lavancement, en deversant dans la heche des amions entiers denrochements. En fait, dans l'hypothese d'une méthode de ce lype el pour une breche importante, on arrive rapidement a à un état de choses difficilement surmontable. En efrel, lobstruction de la digue augmentant la déniveléc totale (niveau dans la rivière - niveatu dans la plaine), quand on arrive près de l'obstruction finale, les vitesses de leau peuvent êlre très considérables au point de rendre problématique toute fermeture lolate.

Ces diffeultés ont dé clairement mises en lumière à propos de quelques-unes des brèches incomparablement moins importantes que celle de Palluel - - auxquelles on dut faire face apris la crue de juin 1948 .

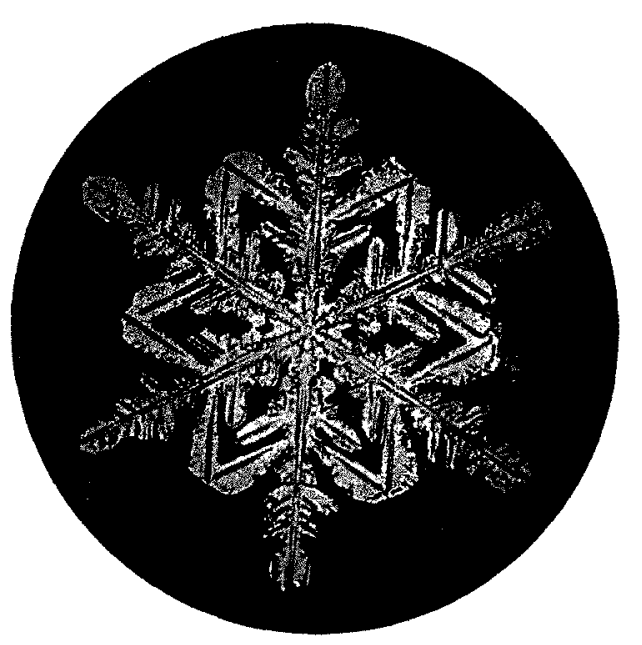

